\title{
Transparency Culture and Financial Communication
}

\author{
Daniela M. Salvioni*
}

\begin{abstract}
The inter-company relationships are affected significantly by the information flows activated by the various players. The fact that a large number of relationships can be essentially ascribed to the sharing of part of the financial risk highlights the crucial role of financial communication.

Financial communication can be an intangible asset of primary importance for corporate development. The achievement of such a goal requires the revision of rules and conduct to obtain messages in true accordance with proper financial corporate principles of truthfulness, clarity, and especially, transparency.

Transparency is an essential requirement for the continuous refining of the convergence between management and information content, and the clarity of the documents periodically transmitted outside. Transparency should facilitate the establishment of a unitary, consistent system of conduct and messages focused on corporate financial dynamics.
\end{abstract}

Keywords: Corporate Governance; Financial Communication; Transparency Culture; Stakeholder Relation

\section{Financial Communication and Inter-Company Relationships}

Corporate systems are at the centre of a system of expectations that, at the same time, promote and guide their behaviour. The satisfaction of expectations makes it possible to set up positive relationships between a company and its business partners and characterizes the prerequisites for the lasting operation of all business initiatives. Expectations become priority goals and management objectives, variously correlated to phenomena of wealth creation and consensus management.

Indeed, the converging interests in corporate reality are wide and diverse, and involve competitive, social and financial spheres. The potential for beneficial interactions with the various classes of stakeholders (contributors of risk and credit capital, workforce, customers, suppliers, financial analysts, etc.) therefore are of a composite character, even if they can all be concisely ascribed to the achievement of satisfactory financial results while adhering to the essential conditions for long-term survival, independence, social fairness and environmental protection.

* Full Professor of Business Administration, University of Brescia (salvioni@eco.unibs.it)

Salvioni Daniela M., Transparency Culture and Financial Communication, Symphonya. Emerging Issues in Management (symphonya.unimib.it), n. 2, 2002, pp. 22-33 
The potential for maintaining corporate success is thus influenced by: the right perception of expectations related to the various players and their relationships, a clear awareness of the resources and skills (technical, organizational, commercial, competitive, etc.) dynamically available, the knowledge of the variables and external factors variously able to influence business and results, the active orientation of all management conduct according to principles of efficacy and efficiency, the ability to transmit messages facilitating knowledge of the degree of expectation satisfaction both actual and projected - and the efforts made in such a direction, and the ability to set up trust-based relationships and manage external consensus.

The constant trend toward corporate global efficacy ${ }^{1}$ highlights the need to set up suitable information sources, able to facilitate, among other things: perception of the various expectations related to the business, an understanding of the constraints and internal and external influencing factors on which the development of the various organizational structures should be rationally based, selection of effective interaction models aimed at constructively combining tangible and intangible components. For example, in profit-oriented companies, the dominant expectations concern the return on and growth of risk capital over time. These are concise parameters, necessarily associated with the adequate satisfaction of several competitive, social and financial conditions.

The need to maintain efficacy stimulates increased attention on external relationships and the functional connections between expectations, management goals, conduct and results. Accordingly, in the current economic context, successful companies show clear signs of integration between corporate governance and management orientation for performance optimisation.

Market globalisation and ever-shorter time scales for assessment processes emphasize the importance of communication and how communication takes place to activate positive relationships between the company and the environment (Figure 1). Communication phenomena of particular significance are particularly significant, aimed at transmitting information concerning the degree of expectation satisfaction to the various recipients.

Well-balanced corporate development depends on the availability of resources adequate to requirements, and optimal resource exploitation to generate results. Assigning available resources to the establishment of structural assets consistent with the business characteristics and operations is instrumental to the preservation of efficacy requirements. Communication facilitates the correct orientation of internal conduct and the management of external consensus management in relation to events.

Resources, business activities and results influence one another. Performance optimisation is defined by the managerial skills progressively developed by the various parts of the organization. In this situation, management efficacy is influenced by: suitable development of knowledge and learning processes to ensure full awareness of the financial, competitive and social implications characterizing the business; the ability to activate suitable information transfer processes to and from the relevant environment. 
Figure 1: Communication and Corporate Efficacy

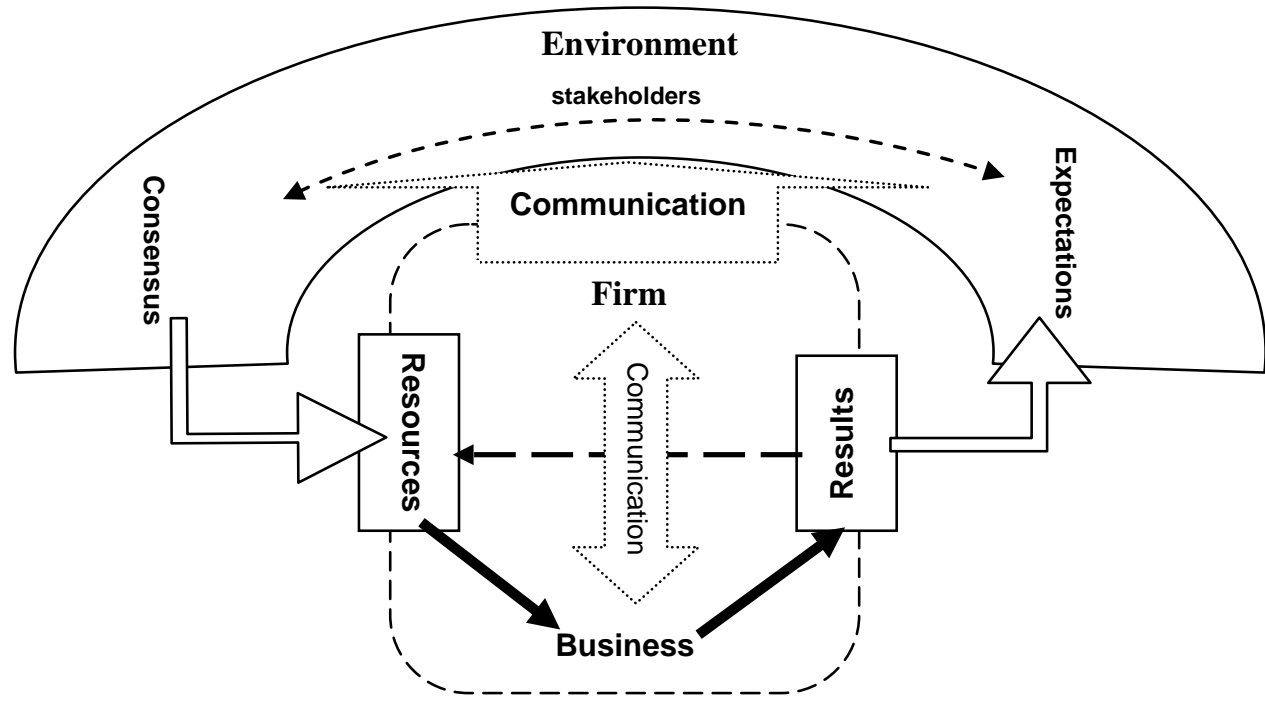

The company's ability to achieve the necessary consensus and the related resources thus also requires:

- distribution of communication aimed at facilitating the completion of assessment processes;

- suitable selection of content, timing and mode of transmission of messages depending on the information expectations of the various types of recipients.

Indeed, corporate efficacy derives from conduct adopted dynamically in the development of management activities, but has essential prerequisites in the distribution of internal and external information. The former is primarily directed at the rationalization of behaviour for the achievement of management goals. The latter is instead aimed at the satisfaction of knowledge and assessment expectations held by the various classes of stakeholders.

In all communication processes aimed at the preservation of conditions for lasting efficacy, a central role is held by the messages with financial content that should be a summary of the actual and expected modes of wealth creation.

With financial measurement tools a company communicates the degree of efficacy and efficiency achieved in management through relevant, concise and selective information designed to symbolically describe complex phenomena that are symptomatic of a large number of spatial and temporal interrelations. Financial communication thus tends to assume primary relevance for internal behavioural trends, and also for the management of external consensus to obtain quantitatively and qualitatively suitable resources for the satisfaction of management needs.

The belief that inter-company relationships are affected significantly by the information flows activated by the various players has become established progressively. The fact that a large number of relationships can be essentially ascribed to the sharing of part of the financial risk highlights the crucial role of financial communication. On the other hand, such a role is amplified by the spread of market and information globalisation phenomena, by the development of information and communication technology, and also by the need to restore validity: this last need is motivated by the insurgence of corporate conduct often 
dominated by personal interests, or the interests of restricted groups, rather than by principles of social equity ${ }^{2}$.

\section{From a Culture of Reticence to a Culture of Transparency}

Financial communication has been studied in depth for many years by scholars of economics and corporate disciplines ${ }^{3}$. In particular, the following topics have been the subject of specific treatment: inter-dependency between communication and exploitation of corporate potential, basic efficacy principles of the distributed messages, also in relation to the existing constraints, behavioural models closely adhering to principles of cost/result ratio optimisation.

Compared to what a significant theoretical study could achieve, the communication actually activated by companies is still lacking in terms of both content and extent. This phenomenon became particularly evident at the beginning of this century with corporate financial disasters that came without warning signs from the quantitativefinancial information divulged previously. Messages with financial content must often be drafted in accordance with constraints and advice of a legal or regulatory nature ${ }^{4}$. Indeed, there are obligations concerning content and publication with minimal requirements, often characterized by varying degrees of peremptoriness depending on the juridical form, the mode of dealing with risk capital, the industrial sector and company size.

Regulatory requirements leave an ample margin of discretion however, and thus the exhaustiveness and completeness of the information contained in published documents are significantly influenced by the goals pursued by each company through this form of communication. In this sense, it is possible to make a distinction between:

- mandatory disclosure deriving from the regulatory constraints aimed at protecting third parties and ensuring the release of information to stakeholders;

- voluntary disclosure offered by companies to complete and extend mandatory disclosure.

However, the existence of constraints does not of itself ensure the quality of information. This is, instead, influenced significantly by the chosen attitude of each company towards consensus management. In particular, the efficacy of financial disclosure, both mandatory and voluntary, reflects the dominant cultural characteristics. Currently, the prevalence of a culture of reticence, often complemented by clearly manipulative policies, determines a generalized lack of trust and interest in quantitative-financial corporate statements. In other words we have the frequent occurrence of vicious circles in which: the lack of willingness to communicate the main corporate financial phenomena limits the ability to satisfy the information expectations of the environment. The inability to satisfy such expectations, in turn, further erodes trust in the communication process, and thus further reduces communication efficacy.

An analysis of the financial information published by companies shows, in fact, that disclosure content is often determined by the will not to communicate, or is manipulated with the aim of establishing relationships with specific sectors of the public. In particular, an analysis of the brief financial statements most frequently published by companies instead shows: 
- communication strategies essentially dictated by the need to fictitiously uphold growth in company value (for example: the inclusion of non-existent or unlikely revenues or the adoption of financial embellishment to represent an equilibrium situation that is better than reality, etc.);

- financial statements expressly aimed at legitimising results aimed at ensuring the lowest possible fiscal drain (often by inflating financial year costs or delaying revenues beyond the relevant period, etc.).

These actions give rise to communication that are far below minimal transparency and fairness requirements for disclosures, that is, the clear correspondence between events and messages transmitted by companies and the fair reconciliation of all expectations expressed by recipients.

Even in the absence of manipulative conduct, in the best of explanations, external financial communication are affected by indiscriminate confidentiality. In these cases, the excessive grouping of asset classes and the strict adherence to minimal regulatory requirements tend to strongly limit transparency in the messages transmitted. In the same way, the use of distribution channels aimed at limiting the spread of information reduces the number of recipients of the disclosure.

The efficacy of financial communication, of which the financial statement is still the most common form, is thus heavily undermined. The awareness that all information transmitted externally contributes to qualifying the corporate image and to creating the potential to acquire or lose consensus is hardly able to establish itself. The widespread critical attitude by recipients persists as a consequence of the often-limited quality of communication.

The need to restore the validity of financial communication is therefore evident. Prevalent attitudes must be changed, by restoring the value of shared general principles (truthfulness, clarity, precision, fairness, completeness of information, prudence, comparability, meaningfulness, etc.) - principles often cited but as often disregarded, precisely because of the limited spread of transparency values.

The pursuit of widespread transparent conduct and the need to revitalize the capital markets have, however, changed over recent years, the quantitative and temporal character of mandatory disclosures in specific operating conditions. Thus for example, the bodies in charge of protecting third parties concerning companies listed on the stock exchange have introduced a quantitative increase and a reduction in frequency (half-year, quarterly) for financial disclosures. The increase in the amount of information made available to the outside world and the shorter time intervals between disclosures have allowed for the overcoming of limits in annual statements related to their historical nature. But at the same time they often induce a focus on the short term or extremely short term, in marked contrast with the corporate system need to be lasting and develop over time.

In summary, the subject of external corporate financial communication is much debated. Many changes are taking place, but we are still very far from the establishment of effective and widely shared conduct models. On the other hand, awareness of the need to satisfy the information requirements of large classes of recipients and the clash between information manipulation and the basic principles of business ethics is growing in importance. Moreover, legal and regulatory constraints are increasingly perceived as determining the spread of information that, while not specifically included in the realm of communications, contribute to the corporate image. 
Therefore, it is time to gradually replace the culture of reticence with the culture of transparency. This is indeed the only way to make the inspiring principle of mandatory disclosure actually operational, and to respect, in voluntary disclosures, the basic tenets of financial propriety.

\section{Requisites for Effective Financial Communication}

The topics of governance, corporate ethics, relationships with stakeholders, transparency and communication efficacy are currently the centre of a wide, complex technical and scientific debate. Almost all of the studies on the subject tend to attribute significant importance to financial communication and its relationship with the potential for success and value creation for the company originating the communication. Often though, attention is focused on the constraint factors and the message content, with limited interest in the satisfaction of expectations and compliance with requirements ensuring effectiveness of messages for the various types of recipients.

At this point it seems appropriate to consider the requirements for effective financial communication, that is the conditions for the establishment of positive relationships between issuer and recipient of the message. The simultaneous presence of two components becomes significant in this context:

- awareness of the role of disclosure and its inclusion in a comprehensive communication policy based on transparency on the issuer's part;

- a positive attitude toward communication reception and consensus on the part of the various groups of recipients.

Awareness of the role of financial communication in the establishment of lasting trusting relationships and constructive external interactions is related in the first place to the well thought out application of regulatory constraints to information transfer objectives. Therefore, mandatory disclosure must be integrated into an intrinsically valid communication program: consistent with a company's positioning in the environment and its fundamental strategic orientation, and complete with respect to the phenomena able to influence attitudes towards corporate values.

Devising the right guidelines for communication choices requires the establishment of a culture aimed at constant quality improvements in disclosures, according to a plan based on valid consensus management models, and characterized by transparency and communication completeness.

Regulations should not be the sole promoters of external financial communication. Such communication should instead be characterized by the goal of constantly satisfying knowledge and assessment expectations, and meet the necessary requirements of ethics and propriety. Specifically, the choice of the defining character of communication from the point of view of content, assessment and distribution requires a suitable combination of constrained, discretionary factors within a communication plan aimed at the concise representation of the elements characterizing corporate financial dynamics.

The spread of a transparency culture represents, as mentioned above, a necessary step to restoring the efficacy of financial communication. Moreover, the value of 
transparency is fundamental in improving internal coordination and ensuring that the various parts of the organization stay on the same wavelength.

After a long period dominated by a limited willingness to transfer the knowledge necessary to develop widespread in-depth assessment processes, the basic principles, values and interpretation mechanisms shared by the ownership, management and the whole organization must return to relationship assumptions between facts, information transmitted and messages issued.

Transparency is an essential requirement for the continuous refining of the convergence between management and information content, and the clarity of the documents periodically transmitted to the external world. Transparency should thus facilitate the establishment of a unitary, consistent system of conduct and messages centred on the constant representation of the phenomena characterizing corporate dynamics.

Recent financial disasters were also due to untruthful communication hiding certain critical factors and thus postponing the emergence of negative financial situations until they were irremediable. This should induce companies to acknowledge the importance of transparency for the improvement of inter-business relationships ${ }^{5}$.

The culture of transparency is also an ethical guideline for all of the people in charge of legitimising the content of financial communication (such as auditors, governance bodies, etc.). The establishment of more transparent conduct in legitimising financial disclosures should indeed increase the value of these institutions in protecting third parties and justify their existence.

Therefore, the culture of transparency tends to directly influence the selection of relevant information from the total available information, always with the necessary completeness and neutrality. It also facilitates internal relationships by establishing:

- consistency with the other forms of communication used by the company to interact with its main audiences;

- exposition clarity and correspondence to the expectations of current and potential recipients;

- significant ability to adjust to the specific requirements of the various partners and contingencies dynamically manifesting themselves;

- information completeness, in general and also, in particular, concerning phenomena rarely taken into account by legal or regulatory constraints, or concerning information relevant to the establishment of constructive interaction with the environment;

- suitable publication of information even via modern information and telecommunication technology.

In particular, the use of telecommunication technology for the distribution of financial information is still very limited.

$\square$ For example, a recent survey of 90 Italian companies listed on the ordinaries part of the stock exchange showed that ${ }^{6}$ only 70 have an Internet site. Only $84 \%$ of these reproduce financial statements on the web site. $98 \%$ of the companies showing their financial statements in the web site show complete statements and $81 \%$ also show the auditor's report. $70 \%$ of the 70 companies also show the consolidated balance 
sheet, complete in 100\% of cases and accompanied by the auditor's report in $88 \%$ of cases. The half-yearly report is included in $65 \%$ of cases, always complete and often together with the auditor's report (63\%). In $71 \%$ of cases, the site includes a whole section on investor relations, while specific information directed at the financial community is present less often. Information on the ownership structure is not always present (structure of the group 60\%, tables on controlled and associated companies 27\%, composition of ownership 39\%, information about management 63\%, tables on extraordinary operations 7\%). Information about market trends is available in $24 \%$ of cases, about corporate strategies in $46 \%$, about corporate history in $81 \%$ and about corporate mission in $86 \%$ of cases. Only $29 \%$ of companies have a specific link for corporate governance, $19 \%$ include meeting regulations, $37 \%$ incorporation documents, $3 \%$ include references to ethical standards and $39 \%$ to a self-regulation code. Finally, only about $2 \%$ of companies with a site include the social balance sheet and the same applies to the environment.

Beside a positive attitude toward communication on the issuer's part, efficacy in financial communication requires a receptive and consensus-oriented attitude by the various external public groups. The actual communication relevance of the financial summary documents for the various recipients is substantially constrained by the:

- dominant socio-cultural factors in the environment in which the target public operates and the value attributed to the disclosure;

- consistency of information with respect to expectations and related perceived reliability;

- the trust-based relationships, both pre-existing and formed through the development of the interaction modes and conduct perceived from the outside.

The recipient attitude is thus related to general conditions (dominant sociocultural factors in the environment and the value attributed to the disclosure) and factors pertaining to the relationship between issuer and recipient of the communication (reliability and consistency of the information with respect to expectations and pre-existing trust-based relationships).

Regarding general conditions, the quest for qualified financial information has grown in importance, due to the importance for many types of recipients of accessing information about corporate financial situations. On the other hand, the reticent and manipulative conduct characteristic of financial information tends to generate an attitude of deep mistrust in potential recipients. Even in this case, however, the spread of a culture of transparency, and its extension to including environmental values, appears to be growing in relevance.

Therefore, sensitivity to financial information and the widespread ability to interpret it do not imply a positive attitude toward it. This latter phenomenon depends instead on the content of the message, the correspondence between communication and expectations and specific relationships based on trust.

Regarding the content of the message, a significant role is undoubtedly played by the regulatory constraints determining its minimal requirements. 
In recent years, the completeness of regulatory provisions concerning the protection of third parties having stakes in corporate results has been heavily discussed; even concerning the existence of national situations characterized by 'civil law' or 'common law' situations. However, almost all studies agree on identifying the way regulatory provisions are applied by companies as the true qualifying factor of financial communication.

The correspondence of information and expectations and related perceived reliability are connected to the attitude assumed by each information issuer toward external communication in general and financial communication in particular. Correspondence to expectations depends, first of all, on global corporate communication policy, on the consensus management model adopted and in the ability and willingness to concisely represent the core parameters. Thus for example, the excessive aggregation of asset classes can make it difficult to discern trends in specific critical success factors.

Reliability is instead related to the potential for judging the merit of the represented financial dynamics and - on first approximation - is ascribable to a significant extent to the determination modes of the values included in the various statements and the underlying disclosure preparation policies. In other words, the disclosure can be considered reliable only if management conduct and the assessment criteria adopted for estimated and conjectured quantities can be verified ${ }^{7}$.

Finally, the trust relationships, both pre-existing and those formed through interaction development and conduct perceived from the outside, depend on all forms of explicit and implicit communication adopted over time. Particular relevance in this context must be ascribed to the intangible components depending on relationships and the information flows that can be inferred from the environment, defining greater or lesser stability in situations of mutual understanding between issuer and recipient of the information. In particular, conduct perceived in the context of specific inter-business relationships, the image and policies of overall transparency and managerial consistency tend to facilitate or obstruct the establishment of trust relationships.

\section{The Validity of Financial Communication}

The validity of financial communication is directly related to the ability to devise concrete, understandable, truthful and exhaustive answers to the demand for information and assessment elements expressed by the various players, and to their willingness to receive the messages available in the environment positively. The players in financial disclosure emphasize its usefulness for all categories of the public interested in analysing changes from different points of view in corporate income, assets and financial equilibrium conditions. In this context it is possible to distinguish between:

- essential expectations, basically shared by all parties interested in financial dynamics;

- $\quad$ specific expectations, expressed by a special type of public and concerning specific types of interactions. 
Essential expectations are related to the assessment of value creation modes. Satisfying these expectations is affected by each company's communication attitude and determines the potential for a positive image and the aggregation of consensus. Specific expectations, instead, are related to special relationships, of significant importance to corporate development, between a company and its environment. These expectations complement the general demand for information and require additional information, often concerned with the in-depth analysis of certain phenomena. Transparency in communication is instrumental in satisfying essential expectations and facilitates the satisfaction of specific expectations.

In this context, the following elements play a significant role: the transmitter of information, the features of the message, the varying interpretation ability of the recipients, feedback, the possible existence of disturbance, amplification phenomena or barriers ascribable to factors interfering with the communication process and possibly limiting or changing its efficacy.

In the current situation of hyper-exposure to information sources and reciprocal influence with the media, interference in corporate communication processes is becoming more and more frequent, with often devastating effects when it is not possible to oppose the interference by resorting to indisputable facts. Thus for example, the prominence recently given to phenomena such as the falsification of accounts, bad faith by some managers, unfaithfulness of auditors in their function of protecting investors, brokers putting personal interest above independence and objectivity, questionable conduct by boards of directors, banks taking part in dubious financial schemes, have all seriously undermined the validity of financial communication.

A limited transparency culture weakens the role of financial communication and also tends to undermine the effectiveness of managerial choices by limiting corporate independence and survival when specific negative perceptions or facts manifest themselves. In reality, financial communication can be an intangible asset of primary importance for corporate development. The achievement of such a goal requires however, the revision of rules and conduct to obtain messages in true accordance with proper financial corporate principles of truthfulness, clarity, and especially, transparency. In the current situation of the obvious inefficacy of messages, financial operators and the media often stress the need for regulatory changes. However, we should not delude ourselves into believing that mandatory disclosure revision would necessarily lead to the essential improvement in communication.

The situation of dissatisfaction that has emerged worldwide has had the effect of aggregating consensus in any case, at least at the level of declarations, about the need to restore the value of transparency to again provide financial communication with the efficacy it needs. However, the main problem is how to infuse the new culture into corporate organizations, and also how to provide for the suitable valuing of the companies that have already adopted it ${ }^{8}$. This path is not easy, since:

- in some companies - mostly large companies listed on stock exchanges - the recent trend toward a quantitative increase in financial communication issued to the external world needs to be overcome while encouraging a mostly qualitative improvement;

- in other companies, mostly of small to medium size, it is instead necessary to abandon the culture of indiscriminate confidentiality in favour of a more significant representation of the actual financial dynamics. 
The effort required by companies is, in any case, amply justified by the benefits that can be obtained, both in terms of better control over inter-business relationships, restoration of a valid image and improvements in the communication cost/benefit ratio.

\section{Notes}

1 'It is possible to define efficacy, in general, as the ability to obtain the desired effects. It is thus possible to identify two fundamental components: on the one hand, the existence of specific expectations concerning certain phenomena and/or conduct; on the other, the propensity to orient phenomena and conduct in such a way as to optimise the methods used to achieve the goals pursued.

That explained above highlights the importance of efficacy as an essential prerequisite to guide a business, given the convergence of multiple interests and the close interdependence between satisfaction of internal and external expectations on one hand, and managerial conduct on the other', D. M. Salvioni, Il sistema di controllo della gestione, Giappichelli, Turin, 1997, pp. 1-2.

${ }^{2}$ Consider the recent American facts for example (Enron, WorldCom, Merck, etc.) and partly also the European ones (Vivendi, etc.), but especially the deep mistrust in corporate communication generated by the prevalence of personal interests, or the interests of specific groups, over correct principles of transparency and social equity.

${ }^{3}$ About this topic see, among others: D. M. Salvioni, Le politiche di comunicazione delle aziende di credito e le informazioni economiche, in La comunicazione nelle banche, Edizioni del Sole 24 Ore, Milan, 1987; V. Coda, La comunicazione economica nella strategia aziendale, in Proceedings of the Cesad Conference, Egea, Milan, 1988; A. Dontoh, Voluntary Disclosure, in Journal of Accounting, Auditing and Finance, no. 4, 1989; D. M. Salvioni, Financial information in global corporate communication process, in 'Economia Aziendale', Vol. IX, no. 2 August 1990; G. Di Stefano, Il sistema delle comunicazioni economico-finanziarie nella realtà aziendale moderna, Giuffrè, Milan, 1990; D. M. Salvioni, Il Bilancio d'esercizio nella comunicazione integrata d'impresa, Giappichelli, Turin, 1992; D. M. Salvioni, Comunicazione, cultura e governo economico d'impresa, Sinergie, no. 43-44, May 1997; Proceedings of the Conference 'Il valore: creazione, misura e comunicazione, Business University L. Bocconi, Milan, 1998; D. M. Salvioni (ed.), L'efficacia della comunicazione economico finanziaria e l'analisi della concorrenza, Giappichelli, Turin, 2002; Aiaf, Creazione di valore e informazione societaria, Quaderno n. 108, Milan, April 2002.

${ }^{4}$ The increased prevalence of large sized companies ('public companies' in the United Kingdom and large 'corporations' in the United States) listed on the stock exchange markets stimulated the regulation of periodic financial disclosures. The first complete regulation was, however, issued only in 1933/34 with the drafting of the well-known United States Federal Laws on Securities and the creation of the Securities Exchange Commission (SEC).

Later English speaking countries promoted initiatives specifically aimed at: the drafting of financial statements based on principles of information and transparency (disclosure), the establishment of forms of control and publicity of financial information, closely connected to the enlargement of the number of shareholders and the public offering of shares, the introduction of statements of supplementary information, the drafting of group consolidated balance sheets, the recourse to certification reports prepared by external independent auditors.

The change of the concept of external corporate financial information in English speaking countries significantly contributed to the spread of regulation mechanisms in other industrialized countries. In particular, rules concerning financial statements were devised in Europe, first at the national level and afterwards coordinated within the European Union through the Community Directives.

5 '...a false or idealized image, or one that is not perfectly consistent with entrepreneurial development, even if able to produce positive effects in the short term, can hardly be characterized by lasting convergence because of the potential progressive dilation of the discrepancy between perception and conduct, or between expectations and results, or also between messages sent to different recipients. 
It must also be remembered that institutional communication has the subscribers of risk capital among its main recipients, that is a significant portion of the financial entities for whose satisfaction the company is established in the first place: toward these parties transparency in management tends to be an ethical imperative.' D. M. Salvioni, Il Bilancio d'esercizio nella comunicazione integrata d'impresa, Giappichelli, Turin, 1992, pp. 50/51.

${ }^{6}$ These are part of the results of a survey of national interest about financial communication and the development of information technology, coordinated by the author and developed by a group of business economy scholars of the University of Brescia. The survey actually did an international comparison of the efficacy of Internet-based financial communication by comparing listed companies in Italy (279 companies), France (80), Germany (80), the United Kingdom (80) and the United States (80)

${ }^{7}$ Actually financial quantities represent values in principle not amenable to manipulation (barring illicit administrative practices). On the contrary, estimated and conjectured values are, by their own nature, constructed and computed based on more or less subjective hypotheses, not always verifiable. Also regulatory provisions leave a sufficiently wide margin of discretion concerning the estimated and conjectured determination that, if correctly interpreted, serves precisely the purpose of differentiating between companies operating in relatively different internal and external contexts.

The assessment of the degree of subjectivity of the information requires subsequent verification through the analysis, also comparative in time and space, of the computational methods adopted for the non-objective values and their interpretation as a function of the characteristics of the company under consideration.

For example, there are companies whose characteristic business actually implies a high incidence of estimated and conjectured values (for example, companies working on large industrial job orders). Of course such a condition does not determine a generalized worsening of the informational function of the financial statements, unless it is impossible to know the method used in the determination, or when the assessment criteria are very approximate and not in full agreement with the representation of the actual financial dynamics.

${ }^{8}$ A primary role in the widespread establishment of a culture of transparency can clearly be played by the media: by emphasizing positive conduct they can act as a stimulus to behavioural changes and the establishment of relationships based on trust. 